\title{
Some Maximal Operators related to Families of Singular Integral Operators*
}

\author{
Jiecheng CHEN \\ Dept of Math, Zhejiang Universitry (Xixi Campus), Hangzhou 310028, P. R. China \\ E-mail: jcchen@mail.hz.zj.cn \\ Dashan FAN \\ Dept of Math, University of Wisconsin-Milwaukee, Milwaukee, WI53201, U.S.A. \\ E-mail: fan@alphal.csd.uwm.edu \\ Meng WANG \\ Dept of Math, Zhejiang Universitry (Xixi Campus), Hangzhou 310028, P. R. China
}

\begin{abstract}
In this paper, we shall study $L^{p}$-boundedness of two kinds of maximal operators related to some families of singular integrals.
\end{abstract}

2000 MSC: Primary 42B20, Secondary 42B25, 42B30

Key words: Maximal operator, Singular Integral, Littlewood-Paley Theory, $L^{p}$-boundedness

*Partially supported by 973 project and NSFZJ 


\section{Introduction}

Let $y^{\prime}$ be a point on the unit sphere $S^{n-1}$ in $R^{n}(n \geq 2)$ and $d \sigma$ be the induced Lebesgue measure on $S^{n-1}$. The Calderón-Zygmund singular integral, initially defined on $S\left(R^{n}\right)$, is defined by

$$
p . v . \int_{R^{n}} b(|y|) \frac{\Omega\left(y^{\prime}\right)}{|y|^{n}} f(x-y) d y,
$$

where $b$ is a measurable function and $\Omega \in L^{1}\left(S^{n-1}\right)$ is a homogeneous function of degree 0 and satisfies $\int_{S^{n-1}} \Omega\left(y^{\prime}\right) d \sigma\left(y^{\prime}\right)=0$. The study and applications of this operator have a long history, for example, see [1], [8]-[11] and their references, etc. In this paper, we are interested in two maximal operators related to this integral. The first operator is defined in $[5]$ by

$$
T(f)(x)=\sup _{h}\left|\int_{R^{n}} h(|y|) \frac{\Omega\left(y^{\prime}\right)}{|y|^{n}} f(x-y) d y\right|
$$

where the supreme is over the set of all radial function $h$ satisfying

$$
\|h\|_{L^{2}\left(R^{+}, \frac{d r}{r}\right)}=\left(\int_{R^{+}}|h(r)|^{2} \frac{d r}{r}\right)^{1 / 2} \leq 1 .
$$

In [5], Chen and Lin proved that if $\Omega \in C\left(S^{n-1}\right)$, then $\|T(f)\|_{p} \leq C_{n, T}\|f\|_{p}$ for $p>\frac{2 n}{2 n-1}$. Also, they pointed out that the range $p>\frac{2 n}{2 n-1}$ is the best possible. However, in this paper, we will prove that the condition $\Omega \in C\left(S^{n-1}\right)$ can be greatly weakened. We will prove the following theorem.

Theorem 1 (i) If $\Omega \in H^{1}\left(S^{n-1}\right)$ and satisfies $\int_{S^{n-1}} \Omega\left(x^{\prime}\right) d \sigma\left(x^{\prime}\right)=0$ where $H^{1}\left(S^{n-1}\right)$ is the Hardy space on $S^{n-1}$, then $\|T(f)\|_{p} \leq C_{n, p, \Omega}\|f\|_{p}$ for all $2 \leq p<\infty$. (ii) If $\Omega \in L^{q}\left(S^{n-1}\right)$ satisfies $\int_{S^{n-1}} \Omega\left(x^{\prime}\right) d \sigma\left(x^{\prime}\right)=0, q \in(1,2]$ and $\frac{2 n q^{\prime}}{n q^{\prime}+2 n-2}<p<\infty$, then $\|T(f)\|_{p} \leq C_{n, p, \Omega}\|f\|_{p}$, where $q^{\prime}$ is the conjugate index of $q$.

Theorem 1 can be extended to the product space. We define

$$
T(f)(x, y)=\sup _{h \in \Delta^{2}}\left|\int_{R^{n} \times R^{m}} \frac{h(|u|,|v|) \Omega\left(u^{\prime}, v^{\prime}\right)}{|u|^{n}|v|^{m}} f(x-u, y-v) d u d v\right|
$$

where $\Delta^{2}$ consists of all $h$ satisfying

$$
\|h\|_{L^{2}\left(R^{+} \times R^{+}, \frac{d r d s}{r s}\right)}=\left(\int_{R^{+} \times R^{+}}|h(r, s)|^{2} \frac{d r d s}{r s}\right)^{1 / 2} \leq 1,
$$


and $\Omega \in L^{1}\left(S^{n-1} \times S^{m-1}\right)$ satisfies the cancellation condition

$$
\int_{S^{n-1}} \Omega\left(u^{\prime}, v^{\prime}\right) d \sigma\left(u^{\prime}\right)=\int_{S^{m-1}} \Omega\left(u^{\prime}, v^{\prime}\right) d \sigma\left(v^{\prime}\right)=0\left(\forall\left(u^{\prime}, v^{\prime}\right) \in S^{n-1} \times S^{m-1}\right) .
$$

We have

Theorem 2 (i) If $\Omega \in L\left(\log { }^{+} L\right)^{2}\left(S^{n-1} \times S^{m-1}\right)$ and satisfies (1), then $\|T(f)\|_{p} \leq$ $C_{n, m, p, \Omega}\|f\|_{p}$ for all $2 \leq p<\infty$. (ii) If $\Omega \in L^{q}\left(S^{n-1} \times S^{m-1}\right)$ and satisfies (1) and $q \in(1,2]$, then $\|T(f)\|_{\gamma} \leq C_{n, m, \gamma, \Omega}\|f\|_{\gamma}$ for $\frac{2 \sigma q^{\prime}}{\sigma q^{\prime}+2 \sigma-2}<\gamma<\infty$, where $\sigma=\min (n, m)$.

Theorem $2(i i)$ has the following generalization. For $\alpha \geq 1$, consider the maximal operator

$$
T^{\alpha}(f)(x, y)=\sup _{h \in \Delta^{\alpha}}\left|\int_{R^{n} \times R^{m}} \frac{h(|u|,|v|) \Omega\left(u^{\prime}, v^{\prime}\right)}{|u|^{n}|v|^{m}} f(x-u, y-v) d u d v\right|,
$$

where $\Delta^{\alpha}$ consists of all $h$ satisfying

$$
\|h\|_{L^{\alpha}\left(R^{+} \times R^{+}, \frac{d r d s}{r s}\right)}=\left(\int_{R^{+} \times R^{+}}|h(r, s)|^{\alpha} \frac{d r d s}{r s}\right)^{1 / \alpha} \leq 1 .
$$

We have

Theorem 3 For $\alpha \in[1,2], q \in(1,2], \Omega \in L^{q}\left(S^{n-1} \times S^{m-1}\right)$ satisfying (1), we have $\left\|T^{\alpha}(f)\right\|_{p} \leq C_{n, m, p, \alpha, \Omega}\|f\|_{p}$ for all $p \in\left(\frac{\alpha^{\prime} \sigma q^{\prime}}{\sigma q^{\prime}+\alpha^{\prime} \sigma-\alpha^{\prime}}, \infty\right)$, where $\sigma=\min (n, m)$.

The second operator we shall consider is

$$
S(f)(x, y)=\sup _{\Omega}\left|\int_{R^{n} \times R^{m}} \frac{h(|u|,|v|) \Omega\left(u^{\prime}, v^{\prime}\right)}{|u|^{n}|v|^{m}} f(x-u, y-v) d u d v\right|
$$

where the supreme is over the set of all $\Omega$ satisfying $\|\Omega\|_{L^{q}\left(S^{n-1} \times S^{m-1}\right)} \leq 1$ and the cancellation condition (1). We have

Theorem 4 For $q>1$ and $q^{\prime} \leq p<\infty, S$ is $L^{p}$-bounded. It also works for the one parameter case. 


\section{Proofs of the Theorems}

\subsection{Proof of Theorem 1}

We follow the argument in [5]. By duality,

$$
T(f)(x)=\left(\sum_{k=-\infty}^{+\infty} \int_{1}^{2}\left|\int_{S^{n-1}} \Omega\left(y^{\prime}\right) f\left(x-2^{k} t y^{\prime}\right) d \sigma\left(y^{\prime}\right)\right|^{2} \frac{d t}{t}\right)^{1 / 2} .
$$

Recalling that $\Omega \in H^{1}\left(S^{n-1}\right)$ has atomic decomposition (see [6]), we may assume that $\Omega\left(y^{\prime}\right)=a\left(y^{\prime}\right)$ is an $H^{1}$-atom which means that $a\left(y^{\prime}\right)$ is an $L^{\infty}$-function satisfying

$$
\begin{aligned}
& \operatorname{supp}(a) \subset\left\{y^{\prime} \in S^{n-1}:\left|y^{\prime}-x_{0}^{\prime}\right|<\rho \text { for some } x_{0}^{\prime} \in S^{n-1} \text { and } \rho \in(0,1]\right\} \\
& \int_{S^{n-1}} a\left(y^{\prime}\right) d \sigma\left(y^{\prime}\right)=0 ; \quad\|a\|_{\infty} \leq \rho^{-(n-1)} .
\end{aligned}
$$

Also, without loss of generality, we assume $x_{0}^{\prime}=\mathbf{1}=(1,0, \cdots, 0)$. Now to prove $(i)$ of the theorem, it suffices to show that

$$
\|T(f)\|_{p} \leq C_{n, p}\|f\|_{p} \text { for } p \geq 2,
$$

where $C_{n, p}$ is a constant independent of atoms $a\left(y^{\prime}\right)$.

Let $\left\{\Phi_{j}\right\}_{-\infty}^{+\infty}$ be a smooth partition of the unit in $(0, \infty)$ adapted to the interval $\left(2^{j-1}, 2^{j+1}\right)$. To be precise, we require the following:

$$
\begin{aligned}
& \Phi_{j} \in C^{\infty}(0, \infty), 0 \leq \Phi_{j} \leq 1, \sum_{j=-\infty}^{+\infty} \Phi_{j}(t)=1 \text { for all } t \in(0, \infty), \\
& \operatorname{supp}\left(\Phi_{j}\right) \subset\left(2^{-j-1}, 2^{j+1}\right) .
\end{aligned}
$$

Define the multiplier operators $S_{j}$ on $R^{n}$ by

$$
\left(S_{j}(f)\right)^{\wedge}(\xi)=\widehat{f}(\xi) \Phi_{j}\left(\left|A_{\rho} \xi\right|\right)
$$

where $A_{\rho}$ is the linear transform such that $A_{\rho} \xi=\left(\rho^{2} \xi_{1}, \rho \xi_{2}, \cdots, \rho \xi_{n}\right)$.

Following the argument on page 123 of [5], we have

$$
T(f)(x)=\sum_{j} T_{j}(f)(x),
$$

where

$$
T_{j}(f)(x)=\left(\sum_{k=-\infty}^{+\infty} \int_{1}^{2}\left|\int_{S^{n-1}} \Omega\left(y^{\prime}\right)\left(S_{k+j} f\right)\left(x-2^{k} t y^{\prime}\right) d \sigma\left(y^{\prime}\right)\right|^{2} \frac{d t}{t}\right)^{1 / 2}
$$


By Plancherel's theorem and Fubini's theorem,

$$
\begin{aligned}
&\left\|T_{j}(f)\right\|_{2}^{2}=\sum_{k=-\infty}^{+\infty} \int_{2^{-(k+j)-1}} \leq\left|A_{\rho} \xi\right| \leq 2^{-(k+j)+1}|\widehat{f}(\xi)|^{2} \\
& \cdot\left\{\int_{1}^{2}\left|\int_{S^{n-1}} \Omega\left(y^{\prime}\right) e^{-i 2^{k} t y^{\prime} \cdot \xi} d \sigma\left(y^{\prime}\right)\right|^{2} \frac{d t}{t}\right\}^{2} d \xi .
\end{aligned}
$$

By page 327 in [8], we know

$$
\begin{aligned}
\int_{1}^{2}\left|\int_{S^{n-1}} \Omega\left(y^{\prime}\right) e^{-i 2^{k} t y^{\prime} \cdot \xi} d \sigma\left(y^{\prime}\right)\right|^{2} \frac{d t}{t} & =\int_{1}^{2}\left|\int_{R} F_{a}(s) e^{-i 2^{k} t s|\xi|} d s\right|^{2} \frac{d t}{t} \\
& =\int_{2^{k}|\xi|}^{2^{k+1}|\xi|}\left|\int_{R} F_{a}(s) e^{-i t s} d s\right|^{2} \frac{d t}{t}
\end{aligned}
$$

where $F_{a}(s)$ satisfies

$$
\begin{aligned}
& \operatorname{supp}\left(F_{a}\right) \subset\left(\xi_{1}^{\prime}-2\left|A_{\rho} \xi^{\prime}\right|, \xi_{1}^{\prime}+2\left|A_{\rho} \xi^{\prime}\right|\right) \\
& \left\|F_{a}\right\|_{\infty} \leq C\left|A_{\rho} \xi^{\prime}\right|^{-1}, \int_{R} F_{a}(s) d s=0, \\
& \text { and } \xi^{\prime}=\frac{\xi}{|\xi|}, A_{\rho} \xi^{\prime}=\left(\rho^{2} \xi_{1}^{\prime}, \rho \xi_{2}^{\prime}, \cdots, \rho \xi_{n}^{\prime}\right) .
\end{aligned}
$$

Thus

$$
\begin{gathered}
\int_{2^{k}|\xi|}^{2^{k+1}|\xi|}\left|\int_{R} F_{a}(s) e^{-i t s} d s\right|^{2} \frac{d t}{t}=\int_{2^{k}|\xi|}^{2^{k+1}|\xi|}\left|\int_{R} F_{a}(s)\left(e^{-i t s}-e^{-i t \xi_{1}^{\prime}}\right) d s\right|^{2} \frac{d t}{t} \\
\leq \int_{2^{k}|\xi|}^{2^{k+1}|\xi|}\left|A_{\rho} \xi^{\prime}\right|^{2} d t \leq C\left(2^{k}|\xi|\left|A_{\rho} \xi^{\prime}\right|\right)^{2}=C\left(2^{k}\left|A_{\rho} \xi\right|\right)^{2}
\end{gathered}
$$

if $2^{k}\left|A_{\rho} \xi\right| \leq 1$. For $2^{k}\left|A_{\rho} \xi\right| \geq 1$, we have

$$
\begin{gathered}
\int_{2^{k}|\xi|}^{2^{k+1}|\xi|}\left|\int_{R} F_{a}(s) e^{-i t s} d s\right|^{2} \frac{d t}{t} \leq C\left(2^{k}|\xi|\right)^{-1}\left\|\widehat{F_{a}}\right\|_{2}^{2} \simeq C\left(2^{k}|\xi|\right)^{-1}\left\|F_{a}\right\|_{2}^{2} \\
=C\left(2^{k}|\xi|\right)^{-1}\left|A_{\rho} \xi^{\prime}\right|^{-1}=C\left(2^{k}\left|A_{\rho} \xi\right|\right)^{-1} .
\end{gathered}
$$

Thus

$$
\begin{aligned}
\left\|T_{j}(f)\right\|_{2}^{2} \leq & C \sum_{k=-\infty}^{+\infty} \int_{2^{-(k+j)-1} \leq\left|A_{\rho} \xi\right| \leq 2^{-(k+j)+1}}|\widehat{f}(\xi)|^{2} \\
& \cdot \min \left\{\left(2^{k}\left|A_{\rho} \xi\right|\right)^{2},\left(2^{k}\left|A_{\rho} \xi\right|\right)^{-1}\right\} d \xi \\
\leq C 2^{-|j|}\|f\|_{2}^{2} . &
\end{aligned}
$$

By page 124 in [5], for $p \geq 2$, there is a function $g$ in $L^{(p / 2)^{\prime}}$ such that $\|g\|_{L^{(p / 2)^{\prime}}}=1$ and

$$
\begin{aligned}
&\left\|T_{j}(f)\right\|_{p}^{2}=\sum_{k=-\infty}^{+\infty} \int_{R^{n}} \int_{1}^{2} \int_{S^{n-1}}\left|\left(S_{k+j} f\right)\left(x-2^{k} t y^{\prime}\right)\right|^{2} \\
& \leq C\|\Omega\|_{1} \sum_{k=-\infty}^{+\infty} \int_{R^{n}}\left|\left(S_{k+j} f\right)\right| d \sigma\left(y^{\prime}\right) \frac{d t}{t}|g(x)| d x\|\Omega\|_{1} \\
& \cdot \int_{1}^{2} \int_{S^{n-1}}\left|\Omega\left(y^{\prime}\right)\right|\left|g\left(x+2^{k} t y^{\prime}\right)\right| d \sigma\left(y^{\prime}\right) \frac{d t}{t} d x \\
& \leq C\|\Omega\|_{1}\left\|\sum_{k=-\infty}^{+\infty}\left|\left(S_{k+j} f\right)\right|^{2}\right\|_{p / 2}\left\|M_{\Omega}(g)\right\|_{(p / 2)^{\prime}}
\end{aligned}
$$


where

$$
\begin{aligned}
M_{\Omega}(g)(x) & =\sup _{k} \frac{1}{2^{k}} \int_{2^{k}}^{2^{k+1}} \int_{S^{n-1}}\left|\Omega\left(y^{\prime}\right)\right|\left|g\left(x+t y^{\prime}\right)\right| d \sigma\left(y^{\prime}\right) d x \\
& \leq \int_{S^{n-1}}\left|\Omega\left(y^{\prime}\right)\right|\left(\sup _{k} \frac{1}{2^{k}} \int_{2^{k}}^{2^{k+1}}\left|g\left(x+t y^{\prime}\right)\right| d x\right) d \sigma\left(y^{\prime}\right) .
\end{aligned}
$$

Thus

$$
\left\|M_{\Omega}(g)\right\|_{(p / 2)^{\prime}} \leq C\|g\|_{(p / 2)^{\prime}} \leq C .
$$

By Littlewood-Paley theorem, we have

$$
\left\|T_{j}(f)\right\|_{p} \leq C\|f\|_{p}
$$

By interpolation, we obtain $(i)$ of Theorem 1.

To prove $(i i)$ of Theorem 1. First, by checking the proof on p.123 in [5], it is easy to see that for $\frac{2 n}{2 n-1}<p \leq 2$, if $\Omega \in L^{2}\left(S^{n-1}\right)$, then

$$
\|T(f)\|_{p} \leq C\|\Omega\|_{2}\|f\|_{p}
$$

However, in the proof of $(i)$, we obtained

$$
\|T(f)\|_{2} \leq C\|\Omega\|_{H^{1}}\|f\|_{2}
$$

So, $(i i)$ follows by interpolation.

\subsection{Proof of Theorem 2}

We will adapt some standard ideas in the one parameter case. By duality, we have

$$
\begin{aligned}
T(f)(x, y)=\left(\sum_{k=-\infty}^{+\infty} \sum_{j=-\infty}^{+\infty} \int_{1}^{2} \int_{1}^{2} \mid \int_{S^{n-1} \times S^{m-1}} \Omega\left(u^{\prime}, v^{\prime}\right)\right. \\
\left.\left.\quad \cdot f\left(x-2^{k} r u^{\prime}, y-2^{j} s v^{\prime}\right) d \sigma\left(u^{\prime}\right) d \sigma\left(v^{\prime}\right)\right|^{2} \frac{d r d s}{r s}\right)^{1 / 2}
\end{aligned}
$$

Take Schwartz functions $p_{1} \in S\left(R^{n}\right)$ and $p_{2} \in S\left(R^{m}\right)$ such that $\operatorname{supp}\left(\widehat{p_{1}}\right) \subset\left\{\frac{1}{2} \leq|x| \leq 2\right\}$, $\operatorname{supp}\left(\widehat{p_{2}}\right) \subset\left\{\frac{1}{2} \leq|y| \leq 2\right\}, 0 \leq \widehat{p_{i}} \leq 1(i=1,2)$ and

$$
\sum_{k=-\infty}^{+\infty}\left|\widehat{p_{1}}\left(2^{k} \xi\right)\right|^{2}=\sum_{j=-\infty}^{+\infty}\left|\widehat{p_{2}}\left(2^{j} \eta\right)\right|^{2}=1 .
$$

Set $\widehat{p_{1, k}}(\xi)=\widehat{p_{1}}\left(2^{k} \xi\right), \widehat{p_{2, j}}(\eta)=\widehat{p_{2}}\left(2^{j} \eta\right)$, and $f_{\mu, \nu}=\left(p_{1, \mu} \otimes p_{2, \nu}\right) *\left(p_{1, \mu} \otimes p_{2, \nu}\right) * f$ where $p_{1, \mu} \otimes p_{2, \nu}(x, y)=p_{1, \mu}(x) p_{2, \nu}(y)$. Then, $f=\sum_{(\mu, \nu) \in \mathbf{Z}^{2}} f_{k+\mu, j+\nu}$ for any $(k, j) \in \mathbf{Z}^{2}$ where 
$\mathbf{Z}$ is the set of all integers. So, by Minkowski's inequality, we have

$$
\begin{aligned}
T(f)(x, y)= & \left(\sum_{k=-\infty}^{+\infty} \sum_{j=-\infty}^{+\infty} \int_{1}^{2} \int_{1}^{2} \mid \sum_{(\mu, \nu) \in \mathbf{Z}^{2}} \int_{S^{n-1} \times S^{m-1}} \Omega\left(u^{\prime}, v^{\prime}\right)\right. \\
\cdot & \left.\left.f_{k+\mu, j+\nu}\left(x-2^{k} r u^{\prime}, y-2^{j} s v^{\prime}\right) d \sigma\left(u^{\prime}\right) d \sigma\left(v^{\prime}\right)\right|^{2} \frac{d r d s}{r s}\right)^{1 / 2} \\
\leq & \sum_{(\mu, \nu) \in \mathbf{Z}^{2}}\left(\sum_{k=-\infty}^{+\infty} \sum_{j=-\infty}^{+\infty} \int_{1}^{2} \int_{1}^{2} \mid \int_{S^{n-1} \times S^{m-1}} \Omega\left(u^{\prime}, v^{\prime}\right)\right. \\
& \left.\left.\cdot f_{k+\mu, j+\nu}\left(x-2^{k} r u^{\prime}, y-2^{j} s v^{\prime}\right) d \sigma\left(u^{\prime}\right) d \sigma\left(v^{\prime}\right)\right|^{2} \frac{d r d s}{r s}\right)^{1 / 2} \\
& \sum_{(\mu, \nu) \in \mathbf{Z}^{2}} T_{\mu, \nu}(f)(x, y) .
\end{aligned}
$$

Now, let

$$
\begin{aligned}
& \Omega_{l}\left(x^{\prime}, y^{\prime}\right)=\Omega\left(x^{\prime}, y^{\prime}\right) \chi_{\Theta_{l}}\left(x^{\prime}, y^{\prime}\right) \\
& \Theta_{0}=\left\{\left(x^{\prime}, y^{\prime}\right) \in S^{n-1} \times S^{m-1}:\left|\Omega\left(x^{\prime}, y^{\prime}\right)\right| \leq 1\right\} \\
& \Theta_{l}=\left\{\left(x^{\prime}, y^{\prime}\right) \in S^{n-1} \times S^{m-1}: 2^{l-1}<\left|\Omega\left(x^{\prime}, y^{\prime}\right)\right| \leq 2^{l}\right\} \text { for } l \geq 1,
\end{aligned}
$$

and

$$
\begin{aligned}
\widetilde{\Omega}_{l}\left(x^{\prime}, y^{\prime}\right)= & \Omega_{l}\left(x^{\prime}, y^{\prime}\right)+\frac{1}{\sigma\left(S^{n-1}\right) \sigma\left(S^{m-1}\right)} \int_{S^{n-1} \times S^{m-1}} \Omega_{l}\left(u^{\prime}, v^{\prime}\right) d \sigma\left(u^{\prime}\right) d \sigma\left(v^{\prime}\right) \\
& -\frac{1}{\sigma\left(S^{n-1}\right)} \int_{S^{n-1}} \Omega_{l}\left(u^{\prime}, y^{\prime}\right) d \sigma\left(u^{\prime}\right)-\frac{1}{\sigma\left(S^{m-1}\right)} \int_{S^{m-1}} \Omega_{l}\left(x^{\prime}, v^{\prime}\right) d \sigma\left(v^{\prime}\right) .
\end{aligned}
$$

It is easy to see that

$$
\begin{aligned}
& \widetilde{\Omega}_{l} \text { satisfies }(1) \text { and } \sum_{l \geq 0} \widetilde{\Omega}_{l}=\Omega \\
& \left\|\widetilde{\Omega}_{l}\right\|_{L^{1}\left(S^{n-1} \times S^{m-1}\right)} \leq 2^{l+2}\left|\Theta_{l}\right| \\
& \left\|\widetilde{\Omega}_{l}\right\|_{L^{\infty}\left(S^{n-1} \times S^{m-1}\right)} \leq 2^{l+2} .
\end{aligned}
$$

Thus

$$
T(f)(x, y) \leq \sum_{l \geq 0} \sum_{(\mu, \nu) \in \mathbf{Z}^{2}} T_{\mu, \nu}^{l}(f)(x, y)
$$

where

$$
\begin{aligned}
T_{\mu, \nu}^{l}(f)(x, y)=\left(\sum_{k, j=-\infty}^{+\infty} \int_{[1,2]^{2}} \mid \int_{S^{n-1} \times S^{m-1}} \widetilde{\Omega}_{l}\left(u^{\prime}, v^{\prime}\right)\right. \\
\\
\left.\left.\quad \cdot f_{k+\mu, j+\nu}\left(x-2^{k} r u^{\prime}, y-2^{j} s v^{\prime}\right) d \sigma\left(u^{\prime}\right) d \sigma\left(v^{\prime}\right)\right|^{2} \frac{d r d s}{r s}\right)^{1 / 2} .
\end{aligned}
$$

Before continuing the proof, we first give two lemmas.

Lemma 5 For

$$
\begin{array}{r}
I_{r, s}^{l}(k, j, \xi, \eta)=\quad \int_{[1,2]^{2}} \mid \int_{S^{n-1} \times S^{m-1}} \widetilde{\Omega}_{l}\left(u^{\prime}, v^{\prime}\right) e^{2 \pi i 2^{k} r u^{\prime} \cdot \xi} \\
\left.\cdot e^{2 \pi i 2^{j} s v^{\prime} \cdot \eta} d \sigma\left(u^{\prime}\right) d \sigma\left(v^{\prime}\right)\right|^{2} \frac{d r d s}{r s}
\end{array}
$$


$\exists \alpha \in(0,1)$ such that

$$
I_{r, s}^{l}(k, j, \xi, \eta) \leq C_{n, m, \alpha} \min \left(\begin{array}{c}
2^{l}\left|\Theta_{l}\right|\left|2^{k} \xi\right|, 2^{l}\left|\Theta_{l}\right|\left|2^{j} \eta\right|, 2^{l}\left|\Theta_{l}\right|\left|2^{k} \xi\right|\left|2^{j} \eta\right|, \\
2^{l}\left|\Theta_{l}\right|, 2^{l}\left|2^{k} \xi\right|^{-\frac{\alpha}{2}}\left|2^{j} \eta\right|, 2^{l}\left|2^{k} \xi\right|\left|2^{j} \eta\right|^{-\frac{\alpha}{2}}, \\
2^{l}\left|2^{k} \xi\right|^{-\frac{\alpha}{2}}, 2^{l}\left|2^{j} \eta\right|^{-\frac{\alpha}{2}}, 2^{l}\left|2^{k} \xi\right|^{-\frac{\alpha}{2}}\left|2^{j} \eta\right|^{-\frac{\alpha}{2}}
\end{array}\right) .
$$

Proof. Similar to the proof of Lemma 10 of [3].

Lemma 6 For $\Omega \in L^{2}\left(S^{n-1} \times S^{m-1}\right)$ and

$$
G=\left\{g_{k, j}\right\}_{k, j} \in L^{p}\left(R^{n} \times R^{m} \rightarrow l^{2}\left(\mathbf{Z}^{2} \rightarrow L^{2}\left([1,2]^{2}, \frac{d r d s}{r s}\right)\right), d x d y\right),
$$

which means that $G$ defines on $R^{n} \times R^{m}$ and takes values in Hilbert space $l^{2}\left(\mathbf{Z}^{2} \rightarrow\right.$ $\left.L^{2}\left([1,2]^{2}, \frac{d r d s}{r s}\right)\right)$ and the Hilbert space consists of all $l^{2}$-sequences defined on $\mathbf{Z}^{2}$ and taking values in $L^{2}\left([1,2]^{2}, \frac{d r d s}{r s}\right)$, there holds

$$
\begin{aligned}
& \|\|\|\mathcal{I}(G)\|_{L^{2}\left([1,2]^{2}, \frac{d r d s}{r s}\right)}\left\|_{L^{2}\left(\mathbf{Z}^{2}\right)}\right\|_{L^{p}\left(R^{n} \times R^{m}, d x d y\right)} \\
& \quad \leq C_{n, m, p}\|\Omega\|_{2}\|\|\|G\|_{L^{2}\left([1,2]^{2}, \frac{d r d s}{r s}\right)}\left\|_{l^{2}\left(\mathbf{Z}^{2}\right)}\right\|_{L^{p}\left(R^{n} \times R^{m}, d x d y\right)},
\end{aligned}
$$

where $p \in(2,2 \min (n, m))$ and

$\mathcal{I}(G)(x, y ; r, s) \stackrel{\text { def }}{=}\left\{\int_{S^{n-1} \times S^{m-1}} \Omega\left(u^{\prime}, v^{\prime}\right) g_{k, j}\left(x+2^{k} r u^{\prime}, y+2^{j} s v^{\prime} ; r, s\right) d \sigma\left(u^{\prime}\right) d \sigma\left(v^{\prime}\right)\right\}_{(k, j) \in \mathbf{Z}^{2}}$.

Proof. We shall use duality method and spherical maximal function. For $p>2$, taking an $f \in L^{(p / 2)^{\prime}}\left(R^{n} \times R^{m}\right)$ such that $\|f\|_{(p / 2)^{\prime}}=1$ and

$$
\begin{aligned}
A & \stackrel{\text { def }}{=}\|\|\|\mathcal{I}(G)\|_{L^{2}\left([1,2]^{2}, \frac{d r d s}{r s}\right)}\left\|_{l^{2}\left(\mathbf{Z}^{2}\right)}\right\|_{L^{p / 2}\left(R^{n} \times R^{m}, d x d y\right)} \\
& =\int_{R^{n} \times R^{m}}\|\| \mathcal{I}(G)\left\|_{L^{2}\left([1,2]^{2}, \frac{d r d s}{r s}\right)}\right\|_{l^{2}\left(\mathbf{Z}^{2}\right)} f(x, y) d x d y
\end{aligned}
$$

Thus, by Hölder's inequality and changes of variables $x$ and $y$, we get

$$
\begin{aligned}
& A= \int_{R^{n} \times R^{m}} f(x, y) \sum_{k, j} \int_{[1,2]^{2}}\left(\int_{S^{n-1} \times S^{m-1}} \Omega\left(u^{\prime}, v^{\prime}\right)\right. \\
&\left.\cdot g_{k, j}\left(x+2^{k} r u^{\prime}, y+2^{j} s v^{\prime} ; r, s\right) d \sigma\left(u^{\prime}\right) d \sigma\left(v^{\prime}\right)\right)^{2} \frac{d r d s}{r s} d x d y \\
& \leq\|\Omega\|_{2}^{2} \int_{R^{n} \times R^{m}} \sum_{k, j} \int_{[1,2]^{2}}\left(\int_{S^{n-1} \times S^{m-1}} f(x, y)\right. \\
& \quad \cdot\left|g_{k, j}\left(x+2^{k} r u^{\prime}, y+2^{j} s v^{\prime} ; r, s\right) d \sigma\left(u^{\prime}\right) d \sigma\left(v^{\prime}\right)\right|^{2} d \sigma\left(u^{\prime}\right) d \sigma\left(v^{\prime}\right) \frac{d r d s}{r s} d x d y
\end{aligned}
$$




$$
\begin{aligned}
& =\|\Omega\|_{2}^{2} \int_{R^{n} \times R^{m}} \sum_{k, j} \int_{[1,2]^{2}}\left|g_{k, j}(x, y ; r, s)\right|^{2} \\
& \quad \cdot \int_{S^{n-1} \times S^{m-1}} f\left(x-2^{k} r u^{\prime}, y-2^{j} s v^{\prime}\right) d \sigma\left(u^{\prime}\right) d \sigma\left(v^{\prime}\right) \frac{d r d s}{r s} d x d y \\
& \leq\|\Omega\|_{2}^{2} \int_{R^{n} \times R^{m}} M_{S}^{\prime} M_{S}^{\prime \prime}(f)(x, y) \sum_{k, j} \int_{[1,2]^{2}}\left|g_{k, j}(x, y ; r, s)\right|^{2} \frac{d r d s}{r s} d x d y \\
& \leq\|\Omega\|_{2}^{2}\left\|M_{S}^{\prime} M_{S}^{\prime \prime}(f)\right\|_{(p / 2)^{\prime}}\left\|\sum_{k, j} \int_{[1,2]^{2}}\left|g_{k, j}(\cdot, \cdot ; r, s)\right|^{2} \frac{d r d s}{r s}\right\|_{p / 2}
\end{aligned}
$$

where

$$
\begin{aligned}
& M_{s}^{\prime}(f)(x, y)=\sup _{r>0} \int_{S^{n-1}}\left|f\left(x-r u^{\prime}, y\right)\right| d \sigma\left(u^{\prime}\right) \\
& M_{s}^{\prime \prime}(f)(x, y)=\sup _{r>0} \int_{S^{m-1}}\left|f\left(x, y-s v^{\prime}\right)\right| d \sigma\left(v^{\prime}\right) .
\end{aligned}
$$

So, by boundedness of spherical maximal function operators, we get

$$
A \leq C_{n, m, p}\|\Omega\|_{2}^{2}\|\|\|G\|_{L^{2}\left([1,2]^{2}, \frac{d r d s}{r s}\right)}\left\|_{l^{2}\left(\mathbf{Z}^{2}\right)}\right\|_{L^{p}\left(R^{n} \times R^{m}, d x d y\right)}^{2}
$$

for $(p / 2)^{\prime}>\max \left(\frac{n}{n-1}, \frac{m}{m-1}\right)$, i.e. $p<2 \min (n, m)$. The last inequality gives the desired result.

Now, we shall prove Theorem $2(i)$.

By Plancherel's identity, we have

$$
\begin{gathered}
\left\|T_{\mu, \nu}^{l}(f)\right\|_{2}^{2}=\int_{R^{n} \times R^{m}} \sum_{k, j} \int_{[1,2]^{2}} \mid \int_{S^{n-1} \times S^{m-1}} \widetilde{\Omega}_{l}\left(u^{\prime}, v^{\prime}\right) \\
\left.\cdot f_{k+\mu, j+\nu}\left(x-2^{k} r u^{\prime}, y-2^{j} s v^{\prime}\right) d \sigma\left(u^{\prime}\right) d \sigma\left(v^{\prime}\right)\right|^{2} \frac{d r d s}{r s} \\
=\sum_{k, j} \int_{[1,2]^{2}} \int_{R^{n} \times R^{m}}\left|\left(f_{k+\mu, j+\nu}\right)^{\wedge}(\xi, \eta)\right|^{2} \mid \int_{S^{n-1} \times S^{m-1}} \widetilde{\Omega}_{l}\left(u^{\prime}, v^{\prime}\right) \\
\left.\cdot e^{2 \pi i 2^{k} r u^{\prime} \cdot \xi} e^{2 \pi i 2^{j} s v^{\prime} \cdot \eta} d \sigma\left(u^{\prime}\right) d \sigma\left(v^{\prime}\right)\right|^{2} d \xi d \eta \frac{d r d s}{r s}
\end{gathered}
$$

Thus, by the definition of $f_{k+\mu, j+\nu}$, we get

$$
\begin{gathered}
\left\|T_{\mu, \nu}^{l}(f)\right\|_{2}^{2} \leq \sum_{k, j} \int_{2^{-(k+\mu)-1} \leq|\xi| \leq 2^{(k+\mu)+1}, 2^{-(j+\nu)-1} \leq|\eta| \leq 2^{-(j+\nu)+1}} \\
\cdot\left|I_{r, s}^{l}(k, j, \xi, \eta)\right|^{2}|\widehat{f}(\xi, \eta)|^{2} d \xi d \eta
\end{gathered}
$$

where $I_{r, s}^{l}$ is defined in (5).

For $p>2$, taking a $g \in L^{(p / 2)^{\prime}}\left(R^{n} \times R^{m}\right)$ such that $\|g\|_{(p / 2)^{\prime}}=1$ and

$$
\left\|\left(T_{\mu, \nu}^{l}(f)\right)^{2}\right\|_{p / 2}=\int_{R^{n} \times R^{m}}\left(T_{\mu, \nu}^{l}(f)(x, y)\right)^{2} g(x, y) d x d y
$$


by Hölder's inequality, we have

$$
\begin{aligned}
&\left\|T_{\mu, \nu}^{l}(f)\right\|_{p}^{2}=\left\|\left(T_{\mu, \nu}^{l}(f)\right)^{2}\right\|_{p / 2} \leq C_{n, m} 2^{l}\left|\Theta_{l}\right| \sum_{k, j} \int_{R^{n} \times R^{m}}\left|f_{k+\mu, j+\nu}(x, y)\right|^{2} \\
& \cdot \int_{[1,2]^{2}} \int_{S^{n-1} \times S^{m-1}}\left|\widetilde{\Omega}_{l}\left(u^{\prime}, v^{\prime}\right) g\left(x+2^{k} r u^{\prime}, y+2^{j} s v^{\prime}\right)\right| \cdot d \sigma\left(u^{\prime}\right) d \sigma\left(v^{\prime}\right) \frac{d r d s}{r s} d x d y \\
& \leq C_{n, m} 2^{l}\left|\Theta_{l}\right| \int_{R^{n} \times R^{m}} \sum_{k, j}\left|f_{k+\mu, j+\nu}(x, y)\right|^{2} M_{\widetilde{\Omega}_{l}}(g)(x, y) d x d y
\end{aligned}
$$

where

$$
\begin{aligned}
M_{\widetilde{\Omega}_{l}}(g)(x, y) & =\sup _{k, j} \int_{[1,2]^{2}} \int_{S^{n-1} \times S^{m-1}}\left|\widetilde{\Omega}_{l}\left(u^{\prime}, v^{\prime}\right) g\left(x+2^{k} r u^{\prime}, y+2^{j} s v^{\prime}\right)\right| d \sigma\left(u^{\prime}\right) d \sigma\left(v^{\prime}\right) \frac{d r d s}{r s} \\
& \leq C_{n, m} \sup _{r, s>0} \frac{1}{r^{n} s^{m}} \int_{\{|x| \leq r\} \times\{|y| \leq s\}}\left|\widetilde{\Omega}_{l}\left(u^{\prime}, v^{\prime}\right) g(x+u, y+v)\right| d u d v .
\end{aligned}
$$

This implies

$$
\left\|M_{\widetilde{\Omega}_{l}}(g)\right\|_{(p / 2)^{\prime}} \leq C_{n, m, p}\left\|\widetilde{\Omega}_{l}\right\|_{1}\|g\|_{(p / 2)^{\prime}} \leq C_{n, m, p} 2^{l}\left|\Theta_{l}\right| .
$$

Thus, by Hölder's inequality and Littlewood-Paley theory, we have

$$
\left\|T_{\mu, \nu}^{l}(f)\right\|_{p}^{2} \leq C_{n, m, p} 2^{2 l}\left|\Theta_{l}\right|^{2}\left\|\sum_{k, j}\left|f_{k+\mu, j+\nu}(x, y)\right|^{2}\right\|_{p / 2} \leq C_{n, m, p} 2^{2 l}\left|\Theta_{l}\right|^{2}\|f\|_{p}^{2},
$$

which shows

$$
\left\|T_{\mu, \nu}^{l}(f)\right\|_{p} \leq C_{n, m, p} 2^{l}\left|\Theta_{l}\right|\|f\|_{p} \quad(p>2)
$$

Now, let

$$
T^{l}(f)(x, y)=\sum_{(\mu, \nu) \in \mathbf{Z}^{2}} T_{\mu, \nu}^{l}(f)(x, y)
$$

we have

$$
\begin{aligned}
\left\|T^{l}(f)\right\|_{p} & \leq \sum_{(\mu, \nu) \in \mathbf{Z}^{2}}\left\|T_{\mu, \nu}^{l}(f)\right\|_{p} \\
& \leq \sum_{(\gamma, \delta) \in \Lambda} \sum_{(\mu, \nu) \in E_{\gamma}^{N} \times E_{\delta}^{N}}\left\|T_{\mu, \nu}^{l}(f)\right\|_{p} \stackrel{\text { def }}{=} \sum_{(\gamma, \delta) \in \Lambda} \Pi_{\gamma, \delta}^{l}(N),
\end{aligned}
$$

where $N$ will be chosen later and large enough, $\Lambda=\{0,1,-1\}, E_{0}^{N}=\{0, \pm 1, \cdots, \pm N l\}$, $E_{-1}^{N}=\{-\infty, \cdots,-N l\}, E_{1}^{N}=\{N l, \cdots,+\infty\}$. For $(\gamma, \delta)=(1,1)$, we have that $\forall(\mu, \nu) \in$ $E_{1}^{N} \times E_{1}^{N}$,

$$
\left\|T_{\mu, \nu}^{l}(f)\right\|_{2} \leq C_{n, m} 2^{l-\mu-\nu}\left|\Theta_{l}\right|\|f\|_{2}
$$


by (7) and (6), thus, by (8) and interpolation, we get

$$
\begin{aligned}
& \left\|T_{\mu, \nu}^{l}(f)\right\|_{p} \leq C_{n, m, p} 2^{l-(\mu+\nu) \theta_{p}}\|f\|_{p}, \\
& \Pi_{1,1}^{l}(N) \leq C_{n, m, p} 2^{l\left(1-N \theta_{p}\right)}\|f\|_{p}
\end{aligned}
$$

for some $\theta_{p} \in(0,1)$. Therefore, for sufficiently large $N$, we have

$$
\sum_{l \geq 0} \Pi_{1,1}^{l}(N) \leq C_{n, m, p}\|f\|_{p} .
$$

Similarly, we have that for sufficiently large $N$ (or see proof in [3]),

$$
\left.\begin{array}{l}
\sum_{l \geq 0} \Pi_{-1,-1}^{l}(N) \leq C_{n, m, p}\|f\|_{p} \\
\sum_{l \geq 0} \Pi_{-1,1}^{l}(N) \leq C_{n, m, p}\|f\|_{p} \\
\sum_{l \geq 0} \Pi_{1,-1}^{l}(N) \leq C_{n, m, p}\|f\|_{p} \\
\sum_{l \geq 0} \Pi_{0,1}^{l}(N) \leq C_{n, m, p} N\|\Omega\|_{L \log (2+L)}\|f\|_{p} \\
\sum_{l \geq 0} \Pi_{1,0}^{l}(N) \leq C_{n, m, p} N\|\Omega\|_{L \log (2+L)}\|f\|_{p} \\
\sum_{l \geq 0} \Pi_{0,-1}^{l}(N) \leq C_{n, m, p} N\|\Omega\|_{L \log (2+L)}\|f\|_{p} \\
\sum_{l \geq 0} \Pi_{-1,0}^{l}(N) \leq C_{n, m, p} N\|\Omega\|_{L \log (2+L)}\|f\|_{p} \\
\sum_{l \geq 0} \Pi_{0,0}^{l}(N) \leq C_{n, m, p} N^{2}\|\Omega\|_{L \log 2(2+L)}\|f\|_{p} .
\end{array}\right\}
$$

By (3), (9) and (10), we get

$$
\|T(f)\|_{p} \leq C_{n, m, p} N^{2}\|\Omega\|_{L \log { }^{2}(2+L)}\|f\|_{p} \quad(p>2)
$$

which proves Theorem $2(i)$.

Now, we shall prove Theorem 2(ii).

By duality, $\exists G=\left\{g_{k, j}\right\}_{(k, j) \in \mathbf{Z}^{2}}$ such that

$$
\|\|\|G\|_{L^{2}\left([1,2]^{2}, \frac{d r d s}{r s}\right)}\left\|_{l^{2}\left(\mathbf{Z}^{2}\right)}\right\|_{L^{p^{\prime}\left(R^{n} \times R^{m}, d x d y\right)}}=1
$$

and

$$
\begin{aligned}
\left\|T_{\mu, \nu}(f)\right\|_{p} \leq \log 2 \int_{R^{n} \times R^{m}} \sum_{k, j}\left\{f_{k+\mu, j+\nu}(x, y)\right. \\
\left.\cdot\left(\int_{[1,2]^{2}}\left|(\mathcal{I}(G))_{k, j}(x, y ; r, s)\right|^{2} \frac{d r d s}{r s}\right)^{1 / 2}\right\} d x d y
\end{aligned}
$$




$$
\begin{aligned}
& \leq \log 2 \int_{R^{n} \times R^{m}}\left(\sum_{k, j}\left|f_{k+\mu, j+\nu}(x, y)\right|^{2}\right)^{1 / 2} \\
& \quad \cdot\left(\sum_{k, j} \int_{[1,2]^{2}}\left|(\mathcal{I}(G))_{k, j}(x, y ; r, s)\right|^{2} \frac{d r d s}{r s}\right)^{1 / 2} d x d y \\
& \leq C_{n, m, p}\|\Omega\|_{2}\left\|\left(\sum_{k, j}\left|f_{k+\mu, j+\nu}(x, y)\right|^{2}\right)^{1 / 2}\right\|_{p},
\end{aligned}
$$

which means that

$$
\left\|T_{\mu, \nu}(f)\right\|_{p} \leq C_{n, m, p}\|\Omega\|_{2}\|f\|_{p} \quad \text { for } p \in\left(\frac{2 \sigma}{2 \sigma-1}, 2\right)
$$

by Littlewood-Paley theory. On the other hand, by Fourier transform, it is easy to show that

$$
\left\|T_{\mu, \nu}(f)\right\|_{2} \leq C_{n, m} 2^{-|\mu|-|\nu|}\|\Omega\|_{2}\|f\|_{2}
$$

So, by interpolation, we get

$$
\|T(f)\|_{p} \leq C_{n, m, p}\|\Omega\|_{2}\|f\|_{p} \quad \text { for } p \in\left(\frac{2 \sigma}{2 \sigma-1}, 2\right) .
$$

Now, for $\Omega \in L^{q}\left(S^{n-1} \times S^{m-1}\right)$ and $1<q \leq 2$, write

$$
T(f)(x, y)=\left\|\tau_{\Omega, r, s}(f)(x, y)\right\|_{L^{2}\left(R^{+} \times R^{+}, \frac{d r d s}{r s}\right)}
$$

where

$$
\tau_{\Omega, r, s}(f)(x, y)=\int_{S^{n-1} \times S^{m-1}} \Omega\left(u^{\prime}, v^{\prime}\right) f\left(x-r u^{\prime}, y-s v^{\prime}\right) d \sigma\left(u^{\prime}\right) d \sigma\left(v^{\prime}\right)
$$

and, set

$$
\tau_{\Omega, r, s}^{z}(f)(x, y)=\int_{S^{n-1} \times S^{m-1}} \widetilde{\Omega}_{z}\left(u^{\prime}, v^{\prime}\right) f\left(x-r u^{\prime}, y-s v^{\prime}\right) d \sigma\left(u^{\prime}\right) d \sigma\left(v^{\prime}\right)
$$

where

$$
\begin{aligned}
\widetilde{\Omega}_{z}\left(x^{\prime}, y^{\prime}\right)= & \Omega_{z}\left(x^{\prime}, y^{\prime}\right)+\frac{1}{\sigma\left(S^{n-1}\right) \sigma\left(S^{m-1}\right)} \int_{S^{n-1} \times S^{m-1}} \Omega_{z}\left(u^{\prime}, v^{\prime}\right) d \sigma\left(u^{\prime}\right) d \sigma\left(v^{\prime}\right) \\
& -\frac{1}{\sigma\left(S^{n-1}\right)} \int_{S^{n-1}} \Omega_{z}\left(u^{\prime}, y^{\prime}\right) d \sigma\left(u^{\prime}\right)-\frac{1}{\sigma\left(S^{m-1}\right)} \int_{S^{m-1}} \Omega_{z}\left(x^{\prime}, v^{\prime}\right) d \sigma\left(v^{\prime}\right) . \\
\Omega_{z}\left(x^{\prime}, y^{\prime}\right)= & \left|\Omega\left(x^{\prime}, y^{\prime}\right)\right|^{\kappa z+\frac{q}{2}} \operatorname{sign}\left(\Omega\left(x^{\prime}, y^{\prime}\right)\right)
\end{aligned}
$$

where $\kappa \in\left(1-\frac{q}{2}, \frac{q}{2}\right)$ is to be determined and $z$ is a complex number. When $\operatorname{Re}(z)=0$, $\widetilde{\Omega}_{z} \in L^{2}\left(S^{n-1} \times S^{m-1}\right)$, by (14), we have

$$
\|\| \tau_{\Omega, r, s}^{z}(f)\left\|_{L^{2}\left(R^{+} \times R^{+}, \frac{d r d s}{r s}\right)}\right\|_{L^{p}\left(R^{n} \times R^{m}\right)} \leq C_{n, m, p}\|\Omega\|_{q}^{q / 2}\|f\|_{p}
$$


for $p \in\left(\frac{2 \sigma}{2 \sigma-1}, 2\right)$. When $\operatorname{Re}(z)=1, \widetilde{\Omega}_{z} \in L^{q /\left(\kappa+\frac{q}{2}\right)}\left(S^{n-1} \times S^{m-1}\right) \subset L\left(\log { }^{+} L\right)^{2}\left(S^{n-1} \times\right.$ $\left.S^{m-1}\right)$, by (11), we have

$$
\|\| \tau_{\Omega, r, s}^{z}(f)\left\|_{L^{2}\left(R^{+} \times R^{+}, \frac{d r d s}{r s}\right)}\right\|_{L^{2}\left(R^{n} \times R^{m}\right)} \leq C_{n, m}\|\Omega\|_{L \log { }^{2}(2+L)}\|f\|_{2} .
$$

Now, for $\gamma \in\left(\frac{2 \sigma q^{\prime}}{\sigma q^{\prime}+2 \sigma-2}, 2\right)$, taking $z_{0}=\left(1-\frac{q}{2}\right) / \kappa \in\left(\frac{2}{q}-1,1\right)$ and $p \in\left(\frac{2 \sigma}{2 \sigma-1}, 2\right)$ such that $\frac{1}{\gamma}=\frac{1-z_{0}}{p}+\frac{z_{0}}{2}$, which is possible because for $\kappa \nearrow \frac{q}{2}$ and $p \searrow \frac{2 \sigma}{2 \sigma-1}$ (where " $\nearrow$ " means "increasingly tends to", "\" means "decreasingly tends to"),

$$
\frac{1-z_{0}}{p}+\frac{z_{0}}{2} \nearrow \frac{\sigma q^{\prime}+2 \sigma-2}{2 \sigma q^{\prime}},
$$

and, for $\kappa \searrow 1-\frac{q}{2}$ and $p \nearrow 2, \frac{1-z_{0}}{p}+\frac{z_{0}}{2} \nearrow \frac{1}{2}$. Noting that $\Omega_{z_{0}}=\Omega$, so, by (15)-(16) and the interpolation of analytic family of operators, we get

$$
\|T(f)\|_{\gamma}=\|\| \tau_{\Omega, r, s}^{z_{0}}(f)\left\|_{L^{2}\left(R^{+} \times R^{+}, \frac{d r d s}{r s}\right)}\right\|_{L^{\gamma}\left(R^{n} \times R^{m}\right)} \leq C_{n, m, \gamma, \Omega}\|f\|_{\gamma}
$$

which proves Theorem $2(i i)$ because the case $\gamma \in[2, \infty)$ is a corollary of Theorem $2(i)$.

\subsection{Proof of Theorem 3}

When $\alpha=1$, by duality and Hölder's inequality, we have

$$
T^{1}(f)(x, y) \leq\|\Omega\|_{q} \sup _{r, s>0}\left(\int_{S^{n-1} \times S^{m-1}}\left|f\left(x-r x^{\prime}, y-s v^{\prime}\right)\right|^{q^{\prime}} d \sigma\left(u^{\prime}\right) d \sigma\left(v^{\prime}\right)\right)^{1 / q^{\prime}} .
$$

So, by the $L^{p}$-boundedness of spherical maximal function (see [11]),

$$
\left\|T^{1}(f)\right\|_{p} \leq C_{n, m, q, p}\|\Omega\|_{q}\|f\|_{p}
$$

for $p>\frac{q^{\prime} \sigma}{\sigma-1}$. On the other hand, for $\alpha=2$, by Theorem 2 ,

$$
\left\|T^{1}(f)\right\|_{p} \leq C_{n, m, q, p}\|\Omega\|_{q}\|f\|_{p}
$$

for $p>\frac{2 \sigma q^{\prime}}{\sigma q^{\prime}+2 \sigma-2}$.

Now, for $\alpha \in(1,2)$ and $H(\cdot, \cdot ; x, y) \in \Delta^{\alpha}$, consider

$$
\begin{array}{r}
T_{H_{z}}(f)(x, y)=\int_{R^{+} \times R^{+}} H_{z}(r, s ; x, y) \int_{S^{n-1} \times S^{m-1}} \Omega\left(u^{\prime}, v^{\prime}\right) \\
\cdot f\left(x-r u^{\prime}, y-s v^{\prime}\right) d \sigma\left(u^{\prime}\right) d \sigma\left(v^{\prime}\right) \frac{d r d s}{r s}
\end{array}
$$


where

$$
H_{z}(r, s ; x, y)=|H(r, s ; x, y)|^{\left(1-\frac{z}{2}\right) \alpha} \operatorname{sign}(H(r, s ; x, y))
$$

and $z$ is a complex number. When $\operatorname{Re}(z)=0,\left\|H_{z}(\cdot, \cdot ; x, y)\right\|_{\Delta^{1}}=\|H(\cdot, \cdot ; x, y)\|_{\Delta^{\alpha}}^{\alpha} \leq 1$, so

$$
\left\|T_{H_{z}}(f)\right\|_{p} \leq\left\|T^{1}(f)\right\|_{p} \leq C_{n, m, q, p}\|\Omega\|_{q}\|f\|_{p}
$$

for $p \in\left(\frac{q^{\prime} \sigma}{\sigma-1}, \infty\right)$. When $\operatorname{Re}(z)=1,\left\|H_{z}(\cdot, \cdot ; x, y)\right\|_{\Delta^{2}}=\|H(\cdot, \cdot ; x, y)\|_{\Delta^{\alpha}}^{\alpha / 2} \leq 1$, so

$$
\left\|T_{H_{z}}(f)\right\|_{p} \leq\left\|T^{2}(f)\right\|_{p} \leq C_{n, m, q, p}\|\Omega\|_{q}\|f\|_{p}
$$

for $p \in\left(\frac{2 \sigma q^{\prime}}{\sigma q^{\prime}+2 \sigma-2}, \infty\right)$. Now, for $f \in C_{c}^{\infty}\left(R^{n} \times R^{m}\right)$, taking $H$ such that $\|H(\cdot, \cdot ; x, y)\|_{\Delta^{\alpha}} \leq$ 1 and

$$
T^{\alpha}(f)(x, y)=T_{H}(f)(x, y) .
$$

Note that for $z_{0}=2\left(1-\frac{1}{\alpha}\right), T_{H_{z_{0}}}(f)=T_{H}(f)$, so, by (17) and (18) and interpolation of analytic family of operators, for $p \in\left(\frac{2 \sigma q^{\prime}}{\sigma q^{\prime}+2 \sigma-2}, \infty\right)$, taking $p_{0} \in\left(\frac{q^{\prime} \sigma}{\sigma-1}, \infty\right)$ and $p_{1} \in$ $\left(\frac{2 \sigma q^{\prime}}{\sigma q^{\prime}+2 \sigma-2}, \infty\right)$ such that $\frac{1}{p}=\frac{1-z_{0}}{p_{0}}+\frac{z_{0}}{p_{1}}$, we have

$$
\left\|T^{\alpha}(f)\right\|_{p}=\left\|T_{H_{z_{0}}}(f)\right\|_{p} \leq C_{n, m, q, p}\|\Omega\|_{q}\|f\|_{p}
$$

which proves Theorem 3 .

\subsection{Proof of Theorem 4}

We shall use the rotation method and only consider the product case. Let

$$
\widetilde{L}^{q}\left(S^{n-1} \times S^{m-1}\right)=\left\{\Omega \in L^{q}\left(S^{n-1} \times S^{m-1}\right): \Omega \text { satisfies }(1)\right\} .
$$

Similarly, we can define $L \widetilde{\log +} L\left(S^{n-1} \times S^{m-1}\right)$ and $L(\widetilde{\log +} L)^{2}\left(S^{n-1} \times S^{m-1}\right)$. Decompose $\widetilde{L}^{q}\left(S^{n-1} \times S^{m-1}\right)$ into four parts

$$
\widetilde{L}^{q}=\widetilde{L}_{o, o}^{q}+\widetilde{L}_{o, e}^{q}+\widetilde{L}_{e, o}^{q}+\widetilde{L}_{e, e}^{q}
$$

where "o" means odd and "e" means even, $\widetilde{L}_{o, e}^{q}$ consists of all $\widetilde{L}^{q}\left(S^{n-1} \times S^{m-1}\right)$ functions which is odd in the first variables and even in the second variables, etc. Say, Refers to [2]. So, we only need to consider the boundedness of

$$
S_{\alpha, \beta}(f)(x, y)=\sup _{\|\Omega\|_{q} \leq, \Omega \in \widetilde{L}_{\alpha, \beta}^{q}}\left|\int_{R^{n} \times R^{m}} \frac{\Omega\left(u^{\prime}, v^{\prime}\right)}{|u|^{n}|v|^{m}} f(x-u, y-v) d u d v\right|
$$


where $\alpha, \beta=o$ or $e$.

For $\alpha=\beta=o$, we have

$$
\begin{aligned}
& \int_{R^{n} \times R^{m}} \frac{\Omega\left(u^{\prime}, v^{\prime}\right)}{|u|^{n}|v|^{m}} f(x-u, y-v) d u d v \\
& \quad=\frac{1}{4} \int_{S^{n-1} \times S^{m-1}} \Omega\left(u^{\prime}, v^{\prime}\right) H_{u^{\prime}} H_{v^{\prime}}(f)(x, y) d \sigma\left(u^{\prime}\right) d \sigma\left(v^{\prime}\right)
\end{aligned}
$$

where

$$
\begin{aligned}
& H_{u^{\prime}}(f)(x, y)=\int_{R} f\left(x-r u^{\prime}, y\right) \frac{d r}{r} \\
& H_{v^{\prime}}(f)(x, y)=\int_{R} f\left(x, y-s v^{\prime}\right) \frac{d s}{s} .
\end{aligned}
$$

By duality,

$$
S_{o, o}(f)(x, y) \leq \frac{1}{4}\left(\int_{S^{n-1} \times S^{m-1}}\left|H_{u^{\prime}} H_{v^{\prime}}(f)(x, y)\right|^{q^{\prime}} d \sigma\left(u^{\prime}\right) d \sigma\left(v^{\prime}\right)\right)^{1 / q^{\prime}}
$$

which means that

$$
\left\|S_{o, o}(f)\right\|_{p} \leq C_{p}\|f\|_{p}
$$

by $L^{p}$-boundedness of $H_{u^{\prime}}$ and $H_{v^{\prime}}$, where $q^{\prime} \leq p<\infty$.

To continue the proof, we need a Lemma from [2]. Let $R_{i}^{\prime}(i=1, \cdots, n)$ and $R_{j}^{\prime \prime}$ $(j=1, \cdots, m)$ denote the $i-t h$ and $j-t h$ Riesz transform on $R^{n}$ and $R^{m}$ respectively, and

$$
\begin{aligned}
& K_{i}^{\prime}(x, y)=R_{i}^{\prime}\left(p \cdot v \cdot \Omega(\cdot, y)|\cdot|^{-n}|y|^{-m}\right)(x) \\
& K_{j}^{\prime \prime}(x, y)=R_{j}^{\prime \prime}\left(p \cdot v \cdot \Omega(x, \cdot)|x|^{-n}|\cdot|^{-m}\right)(y) .
\end{aligned}
$$

Note that the functions in (20) are all homogeneous of order $-n$ in $x$ and homogeneous of order $-m$ in $y$, which means that there are $\omega_{i}^{\prime}, \omega_{j}^{\prime \prime}$ on $S^{n-1} \times S^{m-1}$ such that

$$
\begin{aligned}
& K_{i}^{\prime}(x, y)=\omega_{i}^{\prime}\left(x^{\prime}, y^{\prime}\right)|x|^{-n}|y|^{-m} \\
& K_{j}^{\prime \prime}(x, y)=\omega_{j}^{\prime \prime}\left(x^{\prime}, y^{\prime}\right)|x|^{-n}|y|^{-m} .
\end{aligned}
$$

Define $\widetilde{R}_{i}^{\prime}$ and $\widetilde{R}_{j}^{\prime \prime}$ as follows

$$
\begin{aligned}
& \widetilde{R}_{i}^{\prime}(\Omega)\left(x^{\prime}, y^{\prime}\right)=\omega_{i}^{\prime}\left(x^{\prime}, y^{\prime}\right) \\
& \widetilde{R}_{j}^{\prime \prime}(\Omega)\left(x^{\prime}, y^{\prime}\right)=\omega_{j}^{\prime \prime}\left(x^{\prime}, y^{\prime}\right) .
\end{aligned}
$$

We have

Lemma $7 \widetilde{R}_{i}^{\prime}$ and $\widetilde{R}_{j}^{\prime \prime}$ are bounded from $L \widetilde{\log +} L\left(S^{n-1} \times S^{m-1}\right)$ to $\widetilde{L}^{1}\left(S^{n-1} \times S^{m-1}\right)$, and $\widetilde{R}_{i}^{\prime} \circ \widetilde{R}_{j}^{\prime \prime}$ is bounded from $L(\widetilde{\log +} L)^{2}\left(S^{n-1} \times S^{m-1}\right)$ to $\widetilde{L}^{1}\left(S^{n-1} \times S^{m-1}\right)$, for $i=1, \cdots, n$ and $j=1, \cdots, m$. And, all of them are bounded from $\widetilde{L}^{q}\left(S^{n-1} \times S^{m-1}\right)$ to itself for $q \in(1, \infty)$. 
Now, we continue the proof of Theorem 4 .

For $\alpha=\beta=e$, note that $I=\sum_{i} \sum_{j} \widetilde{R}_{i}^{\prime} \circ \widetilde{R}_{j}^{\prime \prime} \circ \widetilde{R}_{i}^{\prime} \circ \widetilde{R}_{j}^{\prime \prime}$ and $\widetilde{R}_{i}^{\prime} \circ \widetilde{R}_{j}^{\prime \prime}(\Omega) \in \widetilde{L}_{o, o}^{q}\left(S^{n-1} \times S^{m-1}\right)$ for $\Omega \in \widetilde{L}_{e, e}^{q}\left(S^{n-1} \times S^{m-1}\right)$ by Lemma 7 , so

$$
\begin{aligned}
S_{e, e}(f)(x, y) & =\sup _{\|\Omega\|_{q} \leq 1, \Omega \in \widetilde{L}_{e, e}^{q}}\left|\int_{R^{n} \times R^{m}} \frac{\Omega\left(u^{\prime}, v^{\prime}\right)}{|u|^{n}|v|^{m}} f(x-u, y-v) d u d v\right| \\
& =\sup _{\|\Omega\|_{q} \leq 1, \Omega \in \widetilde{L}_{e, e}^{q}}\left|\sum_{i} \sum_{j} \int_{R^{n} \times R^{m}} \frac{\widetilde{R}_{i}^{\prime} \circ \widetilde{R}_{j}^{\prime \prime}(\Omega)\left(u^{\prime}, v^{\prime}\right)}{|u|^{n}|v|^{m}} \widetilde{R}_{i}^{\prime} \circ \widetilde{R}_{j}^{\prime \prime}(f)(x-u, y-v) d u d v\right| \\
& \leq \sum_{i} \sum_{j} \sup _{\|\Omega\|_{q} \leq 1, \Omega \in \widetilde{L}_{e, e}^{q}}\left|\int_{R^{n} \times R^{m}} \frac{\widetilde{R}_{i}^{\prime} \circ \widetilde{R}_{j}^{\prime \prime}(\Omega)\left(u^{\prime}, v^{\prime}\right)}{|u|^{n}|v|^{m}} \widetilde{R}_{i}^{\prime} \circ \widetilde{R}_{j}^{\prime \prime}(f)(x-u, y-v) d u d v\right| \\
& \leq C_{n, m, q} \sum_{i} \sum_{j} S_{o, o}\left(R_{i}^{\prime} \circ R_{j}^{\prime \prime}(f)\right)(x, y)
\end{aligned}
$$

which means that

$$
\left\|S_{e, e}(f)\right\|_{p} \leq C_{n, m, q, p}\|f\|_{p} \quad\left(\text { for } q^{\prime} \leq p<\infty\right)
$$

by (19). Similarly, we have

$$
\begin{array}{rlrl}
\left\|S_{e, o}(f)\right\|_{p} & \leq C_{n, m, q, p}\|f\|_{p} & & \left(\text { for } q^{\prime} \leq p<\infty\right) \\
\left\|S_{0, e}(f)\right\|_{p} \leq C_{n, m, q, p}\|f\|_{p} & & \left(\text { for } q^{\prime} \leq p<\infty\right) .
\end{array}
$$

(19), (21) and (22) give the desired results. 


\section{References}

[1] A. P. Calderón and A. Zygmund, On singular integrals [J], Amer. Jour. Math. 1956, 78:289-309.

[2] J. C. Chen, $L^{p}$ boundedness of singular integrals on product domains [J], Sci. China Ser. A, 2001, 44:681-689.

[3] J. C. Chen and Y. M. Ying, Boundedness of rough singular integrals on product domains [J], submitted.

[4] L. Chen, The maximal operators related to the Calderón-Zygmund method of rotations [J], Ill. Jour. Math. 1989, 33:268-279.

[5] L. Chen and H. Lin, A maximal operator related to a class of singular integrals [J], Ill. Jour. Math. 1990, 34:120-126.

[6] L. Colzani, Hardy space on sphere [M], Ph. D. Thesis, Washington Univ., St. Louis, MO, 1982.

[7] Y. Ding and S. Z. Lu, Homogeneous fractional integrals on Hardy spaces [J], Tôhôku Math. Jour. 2000, 52:153-162.

[8] D. S. Fan and Y. B. Pan, $L^{p}$ boundedness of a singular integral operator [J], Publications Mate. 1997, 41:317-333.

[9] D. S. Fan and Y. B. Pan, Singular integral operators with rough kernels supported by subvarieties [J], Amer. Jour. Math. 1997, 119:799-839.

[10] E. M. Stein, «Singular Integrals and Differentiability Properties of Functions $\gg[M]$, Princeton University Press, Princeton, N.J., 1970.

[11] E. M. Stein, «Harmonic Analysis: Real-variable methods, Orthogonality and Oscillatory Integrals》[M], Princeton University Press, Princeton, N.J., 1993. 